\title{
CARACTERIZACIÓN DE LOS LEPÓRIDOS (MAMMALIA: LEPORIDAE) DEL BOSQUE SECO TROPICAL DEL DEPARTAMENTO DEL TOLIMA (COLOMBIA)*
}

\author{
Román D. Castañeda. PhD. ${ }^{1}$, Carlos E. Viana. MVZ. ${ }^{2}$, Luis A. Buitrago. MVZ. ${ }^{2}$
}

\begin{abstract}
Resumen
Un número de lepóridos del bosque seco tropical del departamento del Tolima (Colombia), no ha sido caracterizado. Con este objetivo se capturaron 22 lepóridos, de los cuales se seleccionaron 13 para realizar estudios morfométricos y citogenéticos. Se tomaron muestras de sangre para determinar el número cromosómico y se evaluaron parámetros morfométricos, entre ellos medidas craneales y corporales. Se encontró en todos los lepóridos un número cromosómico $2 n=38$. Esta información junto con las medidas morfométricas permitieron establecer que dichos lepóridos hacen parte de la especie Sylvilagus brasiliensis, los cuales se han adaptado a las condiciones particulares de la región.
\end{abstract}

Palabras clave: medidas craneales, medidas corporales, número cromosómico, Sylvilagus.

\section{CHARACTERIZATION OF LEPORIDAE (MAMMALIA: LEPORIDAE) IN THE TROPICAL DRY FOREST OF DEPARTMENT TOLIMA (COLOMBIA)}

\begin{abstract}
A number of leporids in the tropical dry forest of Tolima (Colombia) have not been characterized, to this purpose, 22 leporids were captured and 13 of which were selected for morphometric and cytogenetic studies. Blood samples were taken to determine the chromosome numbers and cranial morphometric parameters and body measurements were carried out. All experimental samples had a chromosome number $2 \mathrm{n}=38$. This data together with morphometric measurements allowed to establish that these leporids belong to the genus and specie Sylvilagus brasiliensis which appear to be adapted to the particular environmental conditions of the region.
\end{abstract}

Key words: cranial measurements, body measurements, chromosome number, Sylvilagus.

\footnotetext{
${ }^{*}$ FR: 10-III-2014 FA: 14-XI-2014

${ }^{1}$ Profesor de la Facultad de Medicina Veterinaria y Zootecnia, Universidad del Tolima. Grupo de Investigación en Sistemas Agroforestales Pecuarios. Ibagué, Colombia. E-mail: rcastaneda@ut.edu.co

${ }^{2}$ Grupo de Investigación IMPRONTA. Facultad de Medicina Veterinaria y Zootecnia, Universidad Cooperativa de Colombia sede Ibagué. Ibagué, Colombia. 


\section{INTRODUCCIÓN}

Taxonómicamente los conejos silvestres Sylvilagus hacen parte del orden Lagomorpha, el cual posee dos familias: la Ocotonidae y la Leporidae; esta última, abarca 29 especies de liebres. De acuerdo con CAMPS (2000), se reconocen 24 especies de conejos propiamente dichos enmarcados dentro de 10 géneros diversos. Este es un orden muy particular y diferente a cualquier otro que enmarque una agrupación de roedores; originados en un lepórido común denominador: Alilepus.

En América, solo se reportan lepóridos de los géneros: Sylvilagus (13 especies), Romerolagus (una especie) y Brachilagus (una especie); en nuestra área de estudio, es probable que solo existan individuos del género Sylvilagus o individuos del género Orictolagus (domésticos), que hayan sido asilvestrados. Dentro de los Sylvilagus podría hipotetizarse la presencia de individuos de las especies: brasiliensis, floridanus, y dicei (CAMPS, 2000). Vale la pena destacar, que estos individuos americanos (denominados cottontail, rabo blanco, liebres, o pequeñas liebres) presentan diferentes especies en peligro de extinción, y que en los bosques secos tropicales del valle interandino del alto Magdalena en Colombia, no se reportan trabajos acerca de existencia de sus especies, tamaño de poblaciones relativas, o usos. La especie de conejo de rabo blanco, Sylvilagus brasiliensis, se constituye por 18 subespecies que van desde el centro-este de México hasta el norte de Argentina, y desde el nivel del mar hasta los $4800 \mathrm{~m}$ de altitud (RUEDAS \& SALAZAR-BRAVO, 2007). Los investigadores han criticado la hipótesis de una sola especie politípica generalizada, y sugieren el nombramiento de algunas subespecies como nuevas especies. Sin embargo, las informaciones todavía son escasas. En otros grupos de especies de otras áreas geográficas, el uso de la información cromosómica y los estudios morfológicos han sido importantes para establecer los límites taxonómicos (RUEDAS \& SALAZAR-BRAVO, 2007). Hasta la fecha, sin embargo, los datos cromosómicos y estudios morfológicos disponibles para el género Sylvilagus son limitados. De esta manera, el objetivo de este trabajo de investigación fue caracterizar los lepóridos de bosque seco tropical en el departamento del Tolima, mediante la utilización de métodos morfométricos y citogenéticos.

\section{MATERIALES Y MÉTODOS}

Área de estudio: El trabajo fue realizado en el bosque seco tropical del departamento del Tolima, entre las coordenadas geográficas: 401'06,58" N - 74058'04,31" O y 4³3'56,91" N - 74º57'7,89" O. La región se caracteriza por tener temperaturas superiores a los $24^{\circ} \mathrm{C}$; precipitaciones bimodales durante el año, inferiores a 1400 $\mathrm{mm}$, con mala distribución y alta evaporación; generándose regímenes de humedad secos y subhúmedos. Altura sobre el nivel del mar de 250 a $700 \mathrm{~m}$. El bosque seco tropical se encuentra en la zona central del departamento, bordeando el río Magdalena de sur a norte; donde se incluyen zonas agroecológicas con una biofísica homogénea de piso térmico cálido, y una extensión de 424,97 km cuadrados, con coberturas ralas adensas de pastos, que incluyen áreas de por lo menos 30 municipios del departamento (CHAMORRO \& GALLO, 1998).

Muestreo y análisis: Para determinar las áreas de muestreo se dispuso la información en mapas que fueron sobrepuestos de manera manual. Los espacios de muestreo fueron determinados a partir de sondeos preliminares, encuestas y 
entrevistas exploratorias en cada zona biofísica donde se consideraron las informaciones suministradas por informantes clave de la región. El cruzamiento de las variables permitió la obtención de zonas de vida ubicadas en 5 municipios del departamento del Tolima (Alvarado, Armero-Guayabal, Alpujarra, Ambalema y Valle de San Juan). La recolección de muestras fue realizada a través de trampeos en transectos determinados con jaulas tipo Tomahawk, también fueron realizados muestreos utilizando una red directamente en las madrigueras de los animales. Fueron capturados 22 lepóridos, los cuales fueron llevados al campus de la Universidad Cooperativa de Colombia sede Ibagué, donde se realizó un examen clínico de cada animal encontrándose todos en buenas condiciones de salud. Posteriormente, fueron introducidos en corrales ambientados para su adaptación. Los corrales tuvieron las siguientes dimensiones: largo 2,70 metros por 1,23 metros de ancho y 0,71 metros de alto. Fueron construidos en ladrillo, cemento y techado con teja Eternit y dentro de ellos se recreó un ambiente similar a las condiciones naturales.

El trabajo fue aprobado por el comité de ética de la Facultad de Medicina Veterinaria y Zootecnia de la Universidad Cooperativa de Colombia sede Ibagué. Para realizar los estudios morfométricos y citogenéticos se seleccionaron 13 lepóridos. Los animales fueron identificados por sexo y características particulares. Según los criterios dados en el Manual sobre el cuidado y uso de los animales de experimentación (OLFERT et al., 1998), los animales fueron sedados previamente por vía intramuscular utilizando Clorhidrato de Xilazina al 2\% (Xilazina ${ }^{\star}$ ), posteriormente se administró una sobredosis endovenosa de Tiopental Sódico (Pental ${ }^{\circ}$ ) en una dosis de $200 \mathrm{mg} / \mathrm{kg}$. Previamente se realizó la toma de muestra de sangre (3 a $4 \mathrm{ml}$ ) en la vena yugular, utilizando agujas hipodérmicas número 23G, y tubos Vacutainer ${ }^{\circledR}$ de tapón rojo con Liquemine ${ }^{\circledR}$ (heparina $5000 \mathrm{UI} / \mathrm{ml}$ ). Inmediatamente se colocaron en refrigeración $\left(3-4^{\circ} \mathrm{C}\right)$ en una nevera portátil herméticamente cerrada y fueron trasladadas al Laboratorio de Citogenética Animal de la Facultad de Medicina Veterinaria y de Zootecnia de la Universidad Nacional de Colombia - Sede Bogotá.

Análisis citogenéticos: Para el estudio citogenético se empleó el cultivo de linfocitos de sangre periférica. Las muestras de los 13 lepóridos se tomaron de la vena yugular o de la vena caudal en la base de la cola, en tubos Vacutainer, estériles con $0,1 \mathrm{ml}$ de anticoagulante heparina (5000 UI/ml). Las muestras se transportaron en nevera portátil (4- $6^{\circ} \mathrm{C}$ ), al Laboratorio de Citogenética Animal de la Facultad de Medicina Veterinaria y de Zootecnia de la Universidad Nacional de Colombia sede Bogotá. Para la obtención de cromosomas mitóticos se llevaron a cabo cultivos de linfocitos de sangre periférica de acuerdo a la técnica de MOORHEAD et al. (1960). El protocolo utilizado para la obtención del número cromosómico fue estandarizado por el grupo del Laboratorio de Citogenética de la Universidad Nacional utilizando como medio de cultivo suero fetal bovino, Gentamicina sulfato, L-glutamina y Fitohemaglutinina (mitógeno). Posteriormente obtuvieron las células en metafase, las cuales se llevaron al microscopio para el conteo de los cromosomas.

Análisis morfométricos: Antes de sedar los animales fueron realizadas las medidas corporales LOTO (longitud total), LOCO (longitud de la cola vertebral), LOPA (longitud de la pata trasera), LOOR (longitud de la oreja derecha) utilizando flexómetro digital y PESO g (peso corporal) con balanza digital. Las medidas craneales se tomaron retirando la piel del animal y después del cráneo separándolo de las vértebras cervicales, seguido de esto los cráneos fueron llevados al laboratorio donde fueron introducidos a un horno a temperatura de $180^{\circ} \mathrm{C}$ por un periodo de 
30 minutos con el propósito de deshidratar los músculos. Para retirar los músculos y dejar expuesto y limpio el cráneo para las mediciones se utilizó la técnica de los insectos saprofitos derméstidos, Dermestes lardarius, los cuales estaban alojados en un acuario de vidrio de $50 \mathrm{~cm}$ de largo x $35 \mathrm{~cm}$ de ancho y $30 \mathrm{~cm}$ de alto. Los cráneos fueron dejados a disposición de los derméstidos por un periodo de una semana, tiempo suficiente para dejar los cráneos totalmente limpios; con los cráneos totalmente expuestos, se tomaron las 26 medidas craneales (Tabla 2) utilizando un calibrador vernier digital.

Análisis estadístico: Los resultados fueron analizados utilizando estadística descriptiva; se estimó el promedio de cada medida para machos y hembras de 13 individuos y la desviación estándar de los promedios, para asociar y diferenciar las variaciones respecto a los demás lepóridos ya existentes y poder así clasificarlos taxonómicamente.

\section{RESULTADOS}

Determinación de número cromosómico: Para las hembras se obtuvieron en promedio 132 células en metafase; el 100\% de las células analizadas presentó cariotipo normal con un número modal de $38 \mathrm{XX}$. Para los machos se obtuvieron en promedio 141 células en metafase; el 100\% de las células analizadas presentó cariotipo normal con un número modal de 38 XY (Tabla 1).

Tabla 1. Comparación del número cromosómico de diferentes especies de Sylvilagus (adaptado de RUEDAS \& SALAZAR-BRAVO, 2007).

\begin{tabular}{|c|c|c|c|c|}
\hline Especie & $2 \mathrm{~N}$ & FN & Localidad & Fuente \\
\hline S. audubonii & 42 & 72 & California, USA & $\begin{array}{l}\text { WORTHINGTON \& SUTTON } \\
\text { (1966). }\end{array}$ \\
\hline S. aquaticus & 38 & 72 & Cameron Parish, LA, USA & $\begin{array}{l}\text { ROBINSON et al. (1983), } \\
\text { RUEDAS \& ELDER (1994). }\end{array}$ \\
\hline S. bachmani & 48 & 80 & County, OR, USA & $\begin{array}{l}\text { BENTON \& WORTHINGTON } \\
(1970) .\end{array}$ \\
\hline S. brasiliensis & 36 & 68 & Cuetzalan, Puebla, México & $\begin{array}{l}\text { GÜEREÑA-GÁNDARA et al. } \\
\text { (1983). }\end{array}$ \\
\hline S. brasiliensis & 38 & 72 & Península del Azuero, Panamá & $\begin{array}{l}\text { RUEDAS \& SALAZAR-BRAVO } \\
\text { (2007). }\end{array}$ \\
\hline S. brasiliensis & 40 & 76 & Chiapas, México & $\begin{array}{l}\text { LORENZO \& CERVANTES } \\
\text { (1995). }\end{array}$ \\
\hline S. cunicularius & 42 & $72-74$ & Parres, D.F., México & LORENZO et al. (1993). \\
\hline S. floridanus & 42 & $74-80$ & Connecticut, USA; D.F, México & $\begin{array}{l}\text { HOLDEN \& EABRY (1970), } \\
\text { LORENZO et al. (1993). }\end{array}$ \\
\hline S. graysoni & 42 & 78 & Islas Tres Marías, México & DIERSING \& WILSON (1980). \\
\hline S. nuttallii & 42 & 72 & California, USA & $\begin{array}{l}\text { WORTHINGTON \& SUTTON } \\
\text { (1966). }\end{array}$ \\
\hline S. palustris & 38 & 68 & Halifax County, NC, USA & ROBINSON et al. (1984). \\
\hline S. obscurus & 46 & 84 & Garret County, MD, USA & RUEDAS et al. (1989). \\
\hline S. mansuetus & 48 & 80 & Isla San José, México & CERVANTES et al. (1996). \\
\hline S. transitionalis & 52 & 94 & Mansfield County, CT, USA & $\begin{array}{l}\text { HOLDEN \& EABRY (1970), } \\
\text { RUEDAS et al. (1989). }\end{array}$ \\
\hline S. brasiliensis & 36 & 68 & Estado de Minas Gerais (Brasil) & LANGGUTH \& SOUSA (2008). \\
\hline S. brasiliensis & 38 & & $\begin{array}{l}\text { Departamento del Tolima } \\
\text { (Colombia) }\end{array}$ & Este documento \\
\hline
\end{tabular}


Estudios morfológicos y características de la especie encontrada: Las medidas corporales y craneales son mostradas en la Tabla 2. El peso corporal de los lepóridos fue de $837,87 \pm 84,87$ y $973,80 \pm 88,57 \mathrm{~g}$ para los machos y hembras, respectivamente. Las hembras fueron $15 \%$ más pesadas que los machos.

Tabla 2. Medias $( \pm \mathrm{DE})$ de variables corporales y craneales de lepóridos nativos del trópico bajo en el departamento del Tolima (Colombia).

\begin{tabular}{|c|c|c|}
\hline \multirow[b]{2}{*}{ Características } & \multicolumn{2}{|c|}{ Sylvilagus brasiliensis } \\
\hline & $\begin{array}{c}\text { Machos } \\
\mathrm{n}=9\end{array}$ & $\begin{array}{c}\text { Hembras } \\
\mathrm{n}=4\end{array}$ \\
\hline \multicolumn{3}{|l|}{ Medidas corporales } \\
\hline LOTO (longitud total) & $347,82 \pm 17,11$ & $369,00 \pm 16,73$ \\
\hline LOCO (longitud de la cola vertebral) & $40,05 \pm 4,47$ & $42,34 \pm 1,25$ \\
\hline LOPA (longitud de la pata trasera) & $75,90 \pm 2,92$ & $77,38 \pm 2,96$ \\
\hline LOOR (longitud de la oreja derecha) & $49,26 \pm 2,63$ & $53,69 \pm 2,16$ \\
\hline PESO g (peso corporal) & $837,87 \pm 84,87$ & $973,80 \pm 88,57$ \\
\hline \multicolumn{3}{|l|}{ Medidas craneales } \\
\hline LOMC (longitud mayor del cráneo) & $67,34 \pm 2,15$ & $73,82 \pm 2,19$ \\
\hline PRRO (profundidad del rostro) & $15,01 \pm 1,14$ & $15,87 \pm 0,62$ \\
\hline PRCR (profundidad del cráneo) & $28,32 \pm 1,79$ & $26,99 \pm 1,17$ \\
\hline PRBU (profundidad de la bulla auditiva) & $9,81 \pm 0,83$ & $9,81 \pm 0,18$ \\
\hline LDMX (longitud de hilera de dientes del maxilar) & $12,37 \pm 0,99$ & $13,66 \pm 0,64$ \\
\hline LOBA (longitud basal) & $53,16 \pm 2,71$ & $60,12 \pm 2,15$ \\
\hline LOFI (longitud del foramen incisivo) & $16,87 \pm 1,29$ & $19,27 \pm 1,29$ \\
\hline $\begin{array}{l}\text { ADMX (amplitud a través de la hilera de dientes del } \\
\text { maxilar) }\end{array}$ & $17,13 \pm 2,18$ & $19,79 \pm 0,12$ \\
\hline ANPO (amplitud posdental) & $8,55 \pm 0,30$ & $9,93 \pm 0,20$ \\
\hline ANCC (amplitud de la caja craneana) & $24,58 \pm 0,61$ & $24,77 \pm 0,88$ \\
\hline LOOC (longitud del occipital) & $8,07 \pm 0,54$ & $9,05 \pm 0,16$ \\
\hline LOPP (longitud del puente palatino) & $5,86 \pm 0,86$ & $5,40 \pm 0,27$ \\
\hline LODI (longitud del diastema) & $18,80 \pm 0,85$ & $21,41 \pm 0,81$ \\
\hline AMBU (amplitud de la bulla auditiva) & $26,71 \pm 0,75$ & $28,26 \pm 0,33$ \\
\hline LOFC (longitud del foramen cariótido) & $7,34 \pm 0,81$ & $8,72 \pm 0,27$ \\
\hline ANBO (amplitud del basioccipital) & $8,58 \pm 0,80$ & $9,17 \pm 0,63$ \\
\hline LONA (longitud de los nasales) & $26,36 \pm 3,40$ & $31,10 \pm 0,86$ \\
\hline ANNA (amplitud de los nasales ) & $12,60 \pm 0,55$ & $13,94 \pm 0,30$ \\
\hline ANCI (amplitud del cigomático) & $32,01 \pm 0,82$ & $32,99 \pm 0,05$ \\
\hline ALRM (altura del ramus mandibular) & $12,13 \pm 1,64$ & $12,53 \pm 0,04$ \\
\hline LOMA (longitud de la mandíbula) & $34,65 \pm 1,21$ & $37,38 \pm 0,70$ \\
\hline LDMA (longitud de la hilera de dientes de la mandíbula) & $13,43 \pm 1,53$ & $14,28 \pm 0,37$ \\
\hline ALMA (altura de la mandíbula) & $32,82 \pm 1,78$ & $36,43 \pm 0,85$ \\
\hline ANCO (anchura del canal infraorbital) & $17,83 \pm 2,25$ & $16,65 \pm 0,42$ \\
\hline LOIS (longitud del primer incisivo superior) & $6,95 \pm 1,55$ & $9,47 \pm 2,92$ \\
\hline
\end{tabular}


En la Tabla 3 son mostradas las diferencias de las medidas corporales de los lepóridos de este estudio comparados con otros lepóridos.

Tabla 3. Medidas corporales del S. brasiliensis en el Tolima en comparación con otros grupos de Sylvilagus \pm desviación estándar.

\begin{tabular}{lccccccc}
\hline \multicolumn{1}{c}{ Especies } & $\mathrm{N}^{\circ}$ & $\mathrm{SEX}$ & $\begin{array}{c}\mathrm{PESO} \\
(\mathrm{g})\end{array}$ & $\begin{array}{c}\text { LOTO } \\
(\mathrm{mm})\end{array}$ & $\begin{array}{c}\text { LOPA } \\
(\mathrm{mm})\end{array}$ & $\begin{array}{c}\text { LOOR } \\
(\mathrm{mm})\end{array}$ & $\begin{array}{c}\text { LOCO } \\
(\mathrm{mm})\end{array}$ \\
\hline S. varynaensis $\mathrm{n}$. & 22 & $\mathrm{H}$ & $1739 \pm 41$ & $434 \pm 15,0$ & $86 \pm 2,8$ & $60 \pm 1,7$ & $23 \pm 2,3$ \\
sp. & 23 & $\mathrm{M}$ & $1602 \pm 69$ & $425 \pm 9,0$ & $86 \pm 2,4$ & $61 \pm 1,2$ & $24 \pm 2,6$ \\
& $\mathrm{H}$ & $\mathrm{H}$ & $972 \pm 14$ & $376 \pm 12,0$ & $79 \pm 3,1$ & $59 \pm 3,3$ & $19 \pm 2,2$ \\
S. brasiliensis $\mathrm{B}$. & 19 & $\mathrm{M}$ & $815 \pm 63$ & $350 \pm 16,0$ & $77 \pm 2,7$ & $58 \pm 2,3$ & $18 \pm 2,2$ \\
& 13 & $\mathrm{H}$ & $1180 \pm 31$ & $388 \pm 3,3$ & $79 \pm 0,8$ & $54 \pm 1,1$ & $20 \pm 1,2$ \\
S. brasiliensis $\mathrm{V}$. & 23 & $\mathrm{M}$ & $1010 \pm 34$ & $378 \pm 4,0$ & $76 \pm 0,7$ & $50 \pm 0,5$ & $21 \pm 1,8$ \\
& 20 & $\mathrm{H}$ & $884 \pm 41$ & $362 \pm 7,3$ & $79 \pm 1,5$ & $58 \pm 2,0$ & $21 \pm 2,2$ \\
S. b. meridensis & 22 & $\mathrm{M}$ & $778 \pm 62$ & $343 \pm 11,0$ & $77 \pm 3,3$ & $56 \pm 3,8$ & $19 \pm 1,3$ \\
& 18 & $\mathrm{H}$ & $1233 \pm 52$ & $395 \pm 23,0$ & $81 \pm 2,6$ & $80 \pm 3,8$ & $38 \pm 3,3$ \\
S. f. continentis & 22 & $\mathrm{M}$ & $1115 \pm 42$ & $375 \pm 14,0$ & $81 \pm 1,6$ & $79 \pm 2,2$ & $37 \pm 3,1$ \\
& 16 & $\mathrm{H}$ & $1181 \pm 29$ & $407 \pm 12,0$ & $89 \pm 3,8$ & $57 \pm 2,4$ & $38 \pm 2,5$ \\
S. f. orinoci & 11 & $\mathrm{M}$ & $1197 \pm 91$ & $404 \pm 8,0$ & $84 \pm 3,5$ & $57 \pm 3,6$ & $36 \pm 3,1$ \\
& 40 & $\mathrm{H}$ & $1710 \pm 42$ & $452 \pm 17,0$ & $91 \pm 4,2$ & $61 \pm 2,4$ & $35 \pm 3,6$ \\
S. f. valenciae & 46 & $\mathrm{M}$ & $1469 \pm 38$ & $434 \pm 19,0$ & $91 \pm 3,4$ & $60 \pm 2,6$ & $34 \pm 3,8$ \\
S. brasiliensis. & 4 & $\mathrm{H}$ & $974 \pm 89$ & $369 \pm 16,7$ & $77 \pm 2,96$ & $54 \pm 2,16$ & $42 \pm 1,25$ \\
(Este documento). & 9 & $\mathrm{M}$ & $838 \pm 85$ & $348 \pm 17,11$ & $76 \pm 2,92$ & $49 \pm 2,63$ & $40 \pm 4,47$ \\
\hline
\end{tabular}

\section{DISCUSIÓN}

Determinación de número cromosómico: De acuerdo con LANGGUTH \& SOUSA (2008) los individuos del género Sylvulagus poseen un número diploide de cromosomas que varía de 36 (S. brasiliensis truei) a 52 (S. transitionalis). Los mismos autores determinaron el número cromosómico del $S$. brasiliensis minensis presentando un $2 \mathrm{n}=40$. Entretanto, los lepóridos del género $S$. floridanus poseen 42 cromosomas (PALMER \& ARMSTRONG, 1967). Este trabajo de investigación evidencia y confirma la tendencia descrita por ROBINSON et al. (1984) en la familia Leporidae, en la cual se observa una reducción del número diploide en rearreglos cromosómicos robertsonianos.

Es claro que se evidencia una gran variación en las 21 subespecies del S. brasiliensis, en cuanto al número cromosómico. LANGGUTH \& SOUSA (2008) sugieren que futuramente algunas subespecies tienen que ser consideradas como especies completas. La comparación de nuestros datos con datos ya publicados en investigaciones anteriores que hacen referencia al cariotipo y al número cromosómico de lepóridos pertenecientes a la especie Sylvilagus brasiliensis y otras variedades de Sylvilagus, son mostradas en la Tabla 1. Entretanto, aparentemente la especie de lepórido más cercana a la observada en este trabajo es la reportada por RUE- 
DAS \& SALAZAR-BRAVO (2007), para un conejo encontrado en la península del Azuero en Panamá, el cual tiene 38 cromosomas y fue descrito como $S$. brasiliensis gabbi. Otros lepóridos con el mismo número cromosómico fueron encontrados por ROBINSON et al. (1984) y RUEDAS \& ELDER (1994) en el estado de California (Estados Unidos), el cual fue descrito como S. aquaticus. Asimismo, en el estado de Florida (Estados Unidos) fue reportado por ROBINSON et al. (1984) un lepórido con 38 cromosomas de la especie $S$. palustris. Entretanto, se comparó el número cromosómico de esta especie con el de animales ya clasificados como Sylvilagus floridanus y Sylvilagus brasiliensis, ya que en un estudio de variabilidad genética en la población de lagomorfos de cuatro especies de Sylvilagus se pudo dar a conocer que el Sylvilagus floridanus y el Sylvilagus brasiliensis cuentan con poca diferenciación genética siendo estas las especies más relacionadas entre sí (CERVANTES et al., 1999). De esta manera, se pudo establecer que las subespecies del $S$. brasiliensis poseen menor número de cromosomas (36 a 40) versus las subespecies del $S$. floridanus (42).

Estudios morfológicos y características de la especie encontrada: Analizando la desviación estándar que se realizó a todos los datos obtenidos en las medidas, y correlacionando los resultados morfométricos con el número cromosómico, se determinó que la especie encontrada en el bosque seco tropical del Tolima hace parte de la especie $S$. brasiliensis y que probablemente se trate de una subespecie adaptada a la región. En investigaciones anteriores se encontraron restos arqueológicos de las especies Sylvilagus floridanus ySylvilagus brasiliensis, las cuales fueron tomadas como especies de lepóridos silvestres dentro del bosque del valle del Magdalena tolimense (PEÑA-LEÓN et al., 2007). Sin embargo, en la región de estudio no se encontraron trabajos de investigación evaluando aspectos cromosómicos ni morfométricos de las especies.

La longitud total (LOTO) de los lepóridos encontrados en el bosque seco tropical del departamento del Tolima, fue menor que los $S$. floridanus pero similar a los animales pertenecientes a la especie $S$. brasiliensis, lo cual se demuestra en la Tabla 3.

El peso medio de las hembras encontradas en este estudio fue más liviano que

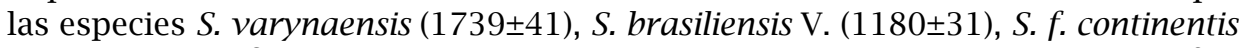
$(1233 \pm 52)$ y $S$. $f$. valenciae $(1710 \pm 42)$. Sin embargo, el peso de esta especie fue muy similar al $S$. b. brasiliensis (Hembra $=972 \pm 14$; Macho $=815 \pm 63$ ). Los únicos lepóridos reportados en la literatura que son más livianos a los reportados en este documento son los $S$. b. meridensis $($ Hembra $=884 \pm 41$; Macho $=778 \pm 62$ ). Entretanto, hubo poca diferencia entre la longitud de la pata (LOPA) y la longitud de la oreja (LOOR) de la especie estudiada con las especies de $S$. brasiliensis. No obstante, la mayor diferenciación entre la especie encontrada en el Tolima y los demás grupos de $S$. brasiliensis estuvo relacionada con la longitud de la cola (LOCO), la cual duplicó en tamaño a los ejemplares reportados como $S$. brasiliensis en otros lugares del mundo, con un promedio de LOCO de 41,2 $\mathrm{mm}$.

\section{CONCLUSIONES}

De acuerdo a los resultados de los análisis cromosómicos, medidas corporales y craneales se establece que los lepóridos presentes en el bosque seco tropical del Tolima son de la especie Sylvilagus brasiliensis y probablemente se trata de una 
subespecie adaptada a las condiciones particulares de la región. La mayor diferencia entre las subespecies de $S$. brasiliensis reportadas en la literatura y la especie encontrada fue el tamaño de la cola, la cual es la mayor cola reportada entre las especies del género Sylvilagus.

\section{AGRADECIMIENTOS}

Los autores agradecen al Comité Nacional para el Desarrollo de la Investigación -CONADI- de la Universidad Cooperativa de Colombia por la financiación de este proyecto. Asimismo, a la Doctora Ligia Mercedes Jiménez Robayo, Directora del Laboratorio de Citogenética Animal de la Universidad Nacional de Colombia sede Bogotá, por toda la colaboración prestada para la realización de los análisis citogenéticos.

\section{BIBLIOGRAFÍA}

CAMPS, J., 2000.- Evolución, y taxonomía de los lepóridos, y el exclusivo origen ibérico del conejo. Lagomorpha, 111: 52-68.

CERVANTES, F.A., RAMÍREZ-SILVA, J.P., MARÍN, A. \& PORTALES, G.L., 1999.- Allozime variation of the cottontail rabbit (Sylvilagus) from Mexico. Z. Säugetierkunde, 64: 357-362.

CHAMORRO, D. \& GALLO, J.E., 1998.- Gramíneas y Leguminosas. Tomas Norato Forero (ed.). El Poira, Ibagué.

LANGGUTH, A. \& SOUSA, M.A., 2008.- The karyotype of Sylwlagus brasiliensis minensis Thomas (Lagomorpha: Leporidae). Revista Nordestina de Biología, 1: 59-62.

MOORHEAD, P.S., NOWELL, P.C., MELLMANW, J., BATTIPSD, T. \& UNGERFORD, D.A., 1960.- Chromosome preparations of leukocytes cultured from human peripheral blood. Experimental Cell Research, 20 (3): 613-616.

OLFERT, E.D., CROSS, B.M. \& MCWILLIAM, A.A., 1998.- Guide to the care and use of experimental animals. Vol. 1. 2nd ed. Canadian Council on Animal Care, Ontario.

PALMER, C. \& ARMSTRONG, R., 1967.- Chromosome number and karyotype of Sylvilagus floridanus, the eastern cottontail. Mammal Chromosome Newsletter, 8: 282-283.

PEÑA-LEÓN, G.A., GÓMEZ-GARCÍA, A.N. \& SALGADO-LÓPEZ, H., 2007.- Restos faunísticos en contextos funerarios prehispánicos del Valle del Magdalena tolimense (Espinal, Colombia). Caldasia, 29: 1-17.

ROBINSON, T.J., ELDER, F.F.B. \& CHAPMAN, J.A., 1984.- Evolution of chromosomal variation in cottontails, genus Sylvilagus (Mammalia: Lagomorpha). Cytogenetic and Genome Research, 38: 282-289.

RUEDAS, L.A. \& ELDER, F.F.B., 1994.- On the putative existence of a diploid number 42 cytotype of Sylvilagus aquaticum (Bachman, 1837) (Lagomorpha: Leporidae). Mammalia, 58: 145-148.

RUEDAS, L.A. \& SALAZAR-BRAVO, J., 2007.- Morphological and chromosomal taxonomic assessment of Sylvilagus brasiliensis gabbi (Leporidae). Mammalia, 71: 63-69. 